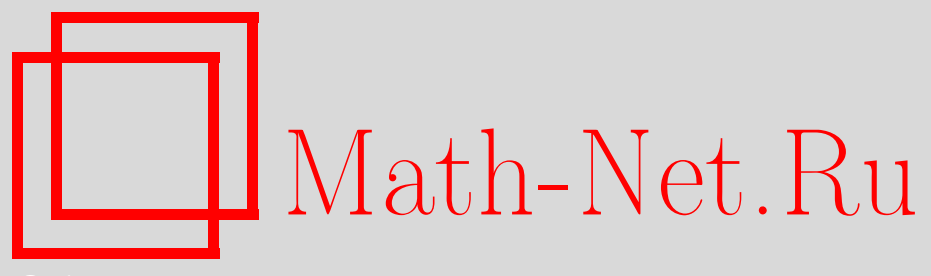

Н. П. Долбилин, О. Р. Мусин, Г. Эдельсбруннер, Об оптимальности функционалов на триангуляциях множеств Делоне, УМН, 2012, том 67, выпуск 4, 189-190

DOI: https://doi.org/10.4213/rm9487

Использование Общероссийского математического портала Math-Net.Ru подразумевает, что вы прочитали и согласны с пользовательским соглашением http://www.mathnet.ru/rus/agreement

Параметры загрузки:

IP : 3.91 .87 .62

26 апреля 2023 г., 15:28:06

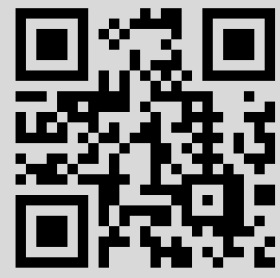




\section{Об оптимальности функционалов на триангуляциях множеств Делоне}

\section{Н. П. Долбилин, О.Р. Мусин, Г. Эдельсбруннер}

В заметке мы рассматриваем плотности функционалов на равномерно ограниченных триангуляциях с заданным множеством вершин. Доказано, что если функционал достигает минимума на триангуляциях Делоне для конечных множеств, то минимум плотности достигается и на бесконечной триангуляции Делоне.

Множеством Делоне называется множество $X \subset \mathbf{E}^{d}$, для которого существуют положительные числа $r$ и $R$ такие, что для любого открытого $d$-шара $B_{r}^{\circ}$ и замкнутого шара $\bar{B}_{R}$ радиусов $r$ и $R$ соответственно выполнены неравенства $\left|B_{r}^{0} \cap X\right| \leqslant 1$ и $\left|\bar{B}_{R} \cap X\right| \geqslant 1$, где $|Y|$ означает мощность множества $Y$. В статье будем рассматривать множества Делоне в общем положении, в том смысле, что никакое подмножество такого множества, состоящее из $d+2$ точек, не лежит на одной $(d-1)$-сфере.

Будем рассматривать семейство симплициальных разбиений (триангуляций) $T$ пространства $\mathbf{E}^{d}$ с фиксированным множеством вершин $X$, где $X$ есть множество Делоне. Если для триангуляции $T$ существует положительное число $q=q(T)$ такое, что для любого $d$-симплекса $S \in T$ радиус $\mathcal{R}(S)$ его описанного шара не превосходит $q: \mathcal{R}(S) \leqslant q$, то будем называть триангуляцию $T$ равномерно ограниченной и обозначать семейство всех таких триангуляций через $\Theta(X)$.

Множества Делоне были введены Б. Н. Делоне (1924 г.) под названием $(r, R)$-система. Он доказал (см., например, работу [1]), что для множества Делоне $X$ существует и притом единственная триангуляция Делоне $D T(X)$, множество вершин которой совпадает с $X$. Эта триангуляция является равномерно ограниченной с $q=R$, т. е. $D T(X) \in \Theta(X)$.

Для любого множества Делоне можно построить триангуляцию, которая не является равномерно ограниченной. Вместе с тем остается открытой проблема (Л. Данцер и М. Бошерницан): верно ли, что для любого множества Делоне на плоскости существует не содержащий точек из этого множества треугольник сколь угодно большой площади?

Рассмотрим функционал $F$, определенный на $d$-симплексах. Мы будем считать, что $F$ непрерывно зависит от параметров симплекса (например, его ребер). Если $X-$ конечное множество и $T$ - триангуляция выпуклой оболочки $\operatorname{conv}(X)$ с множеством вершин в $X$, можно определить функционал на конечной триангуляции $T: F(T)=$ $\sum_{S \in T} F(S)$

Пусть $B_{\alpha}$ - шар радиуса $\alpha$ с центром в начале координат $O$ и $T \in \Theta(X)$. Следующий нижний предел

$$
\bar{F}(T):=\varliminf_{\alpha \rightarrow \infty} \frac{1}{\operatorname{vol}\left(B_{\alpha}\right)} \sum_{S \subset B_{\alpha}} F(S)
$$

назовем (нижней) плотностъю функиионала $F$ на триангуляции $T$.

Лемма. Для любого непрерывного функционала $F$, для множества Делоне $X u$ триангулячии $T \in \Theta(X)$ на плоскости значение $\bar{F}(T)$ конечно.

Теорема. Пусть непрерывный функционал F на множестве всех триангуляиий выпуклой оболочки конечного множества $Y$ на плоскости достигает минимума на триангуляиии Делоне DT(Y). Тогда для любого множества Делоне X на плоскости плотность $\bar{F}$ достигает минимума на $D T(X)$.

Работа выполнена при поддержке гранта Правительства РФ № 11.G34.31.0053, РФФИ (грант № 11-01-00735) и DMS-1101688. 
ДоказАтельство. 1. Пусть $T \in \Theta(X)$ - триангуляция с параметром $q$. Рассмотрим симплициальный комплекс $\tau_{\alpha}(T)$, состоящий из всех $d$-симплексов триангуляции $T$, целиком лежащих в $B_{\alpha}$. Границу $\tau_{\alpha}(T)$ обозначим через $P_{\alpha}(T)$, а множество ее вершин - через $V_{\alpha}(T)$. Пусть $C_{\alpha}$ - выпуклая оболочка множества $V_{\alpha}$. Для $d=2$ дополнение $C_{\alpha} \backslash P_{\alpha}$ состоит из многоугольников. Поскольку любой многоугольник можно триангулировать, не добавляя вершин, то $\tau_{\alpha}$ можно дополнить до триангуляции $\psi_{\alpha}$ выпуклой оболочки $C_{\alpha}$.

2. Для $d=2$ число $K_{\alpha}$ треугольников, принадлежащих кругу $B_{\alpha}$, равно $O\left(\alpha^{2}\right)$. Из этого следует, что число треугольников в $\psi_{\alpha}$ равно

$$
L_{\alpha}=K_{\alpha}+O\left(K_{\alpha}^{(d-1) / d}\right) .
$$

3. Из пп. 1 и 2 следует, что

$$
\lim _{\alpha \rightarrow \infty} \frac{F\left(\tau_{\alpha}\right)}{F\left(\psi_{\alpha}\right)}=1 .
$$

По условию теоремы $F\left(\psi_{\alpha}\right)$ не меньше, чем значение $F$ на триангуляции Делоне для множества вершин триангуляции $\psi_{\alpha}$. Тогда из $(*)$ вытекает требуемое.

ЗАмечАниЕ. Препятствием для многомерного, $d>2$, доказательства является п. 1. Чтобы завершить доказательство для любой размерности, достаточно показать, что $H_{\alpha}:=C_{\alpha} \backslash P_{\alpha}$ можно триангулировать, возможно с добавлением $N$ точек, где число $N$ может быть порядка $O\left(\left|V_{\alpha}\right|^{a}\right), a<d /(d-1)$.

СледствиЕ. Пусть для треугольника $\Delta$ со сторонами $a_{1}, a_{2}, a_{3}$ определены следующие функииональ:

1) $F_{1}(\Delta):=\mathcal{R}^{a}(\Delta)$, где $\mathcal{R}(\Delta)$ - радиус описанной окружсости, $a>0$;

2) $F_{2}(\Delta):=\frac{a_{1}^{2}+a_{2}^{2}+a_{3}^{2}}{s(\Delta)}$, где $s(\Delta)-$ ллощ, адь треугольника;

3) $F_{3}(\Delta):=-\rho(\Delta)$, где $\rho(\Delta)-$ радиус вписанной окружсности;

4) $F_{4}(\Delta):=\left(a_{1}^{2}+a_{2}^{2}+a_{3}^{2}\right) s(\Delta)$

5) $F_{5}(\Delta):=\mathcal{R}^{a}(\Delta) s(\Delta), a \geqslant 1$

6) $F_{6}(\Delta):=\|b(\Delta)-c(\Delta)\|^{2} s(\Delta)$, где $b-$ центр тяжести $\Delta$, , с - иентр описанной окружности.

Тогда их плотности $\bar{F}_{i}, i=1, \ldots, 6$, достигают минимума на триангулячии Делоне.

Заметим, что для конечных множеств оптимальность для функционалов $F_{1}, F_{2}$ была доказана в работе [3], для $F_{3}-$ в [2], для $F_{4}-$ в [5] и для $F_{5}, F_{6}-$ в работе [4].

\section{Список литературы}

[1] Б. Н. Делоне, УМН, 1937, №3, 16-62. [2] Т. Lambert, Proceedings of the 6th Canadian Conference on Computational Geometry (Saskatoon, SK, Canada, 1994), University of Saskatchewan, 1994, 201-206. [3] O.R. Musin, SCG'97 Proceedings of the 13th Annual ACM Symposium on Computational Geometry (Nice, France, 1997), ACM, New York, 1997, 424-426. [4] O.R. Musin, http://delone120.mi.ras.ru/app/Musin.pdf. [5] V. T. Rajan, Discr. Comput. Geometry, 12:2 (1994), 189-202.

Н. П. Долбилин (N. P. Dolbilin)

Математический институт им. В. А. Стеклова РАН

O. P. Мусин (O. R. Musin)

University of Texas at Brownsville, Brownsville, USA

Г. Эдельсбруннер (Н. Edelsbrunner)

Institute of Science and Technology, Vienna, Austria
Представлено В. М. Бухштабером Принято редколлегией 21.06.2012 